\title{
Robust 4-channel WDM demultiplexing filter based on collectively tuned silicon microrings
}

\author{
Peter De Heyn, Michael Vanslembrouck and Dries \\ Van Thourhout \\ Photonics Research Group, Dept. Information Technology \\ Ghent University - imec, B-9000, Gent, Belgium \\ Peter.DeHeyn@Intec.Ugent.be
}

\author{
Joris Van Campenhout, Jeroen De Coster, Marianna \\ Pantouvaki and Philippe Absil \\ IMEC, Kapeldreef 75, Leuven, Belgium
}

\begin{abstract}
We demonstrate a robust 4-channel WDM demultiplexing filter based on cascaded silicon microring resonators. A comparison is made between first- and secondorder filters regarding the robustness of the filter response to wafer-scale fabrication variations.
\end{abstract}

\section{Keywords-microring resonators; WDM; Silicon-on-Insulator}

\section{INTRODUCTION}

Aggregate bandwidth requirements for I/O communication in advanced CMOS nodes are expected to reach the level of multiple $\mathrm{TB} / \mathrm{s}$ by 2018 [1]. Optical interconnects based on silicon photonics are increasingly being considered as a viable alternative to enable further $\mathrm{I} / \mathrm{O}$ scaling [2]. An attractive approach to scale the bandwidth of silicon-based optical interconnects is the adoption of wavelength-division multiplexing (WDM).

One of the major issues for realizing WDM optical devices using a high-index contrast platform such as silicon-oninsulator (SOI) is the sensitivity of these components to variations in waveguide dimensions. These include linewidth variations during lithographic pattern definition as well as thickness variations of the top silicon layer of the SOI stack [3]. In addition, silicon wavelength-selective devices are known to be highly sensitive to temperature variations. These perturbations directly influence the effective refractive index and give rise to a detrimental shift of the resonance wavelength of WDM filters such as microring resonator based filters [4], both on chip level and wafer level [3].

Silicon microring resonators (MRR) can be made very compact, and can be designed with large free-spectral ranges (FSR). Ring-based WDM components have been demonstrated with a considerable smaller footprint in comparison to other filter technologies such as arrayed waveguide gratings (AWG) or echelle gratings [5]. Higherorder rings give more freedom in comparison with first-order rings in designing the filter specifications regarding crosstalk to neighboring channels, insertion loss and $3 \mathrm{~dB}$ bandwidth. With higher-order filters, one can achieve flat-top behavior and increased $3 \mathrm{~dB}$ bandwidth for the same crosstalk [6]. Besides enabling high integration densities, this compactness also enables low-power thermal tuning. For a WDM receiver, individual heaters on each filter are typically used to thermally tune the resonances onto the laser grid, compensating for fabrication non-uniformity and thermal variations. WDM demultiplexers with multiple channels have been demonstrated using single and higher-order MRRs with such individual channel tuning [7-8]. In particular for these higher-order filters, the thermal tuning algorithms can be very complex and will substantially add to system complexity. On the other hand, collective thermal tuning is a potentially much easier approach to compensate for ambient temperature fluctuations, as it only involves the control of a single heating element. However, in that case, a robust filter design is needed which can compensate for the fabrication non-uniformities. In addition, the filter should be sufficiently compact

To make waveguides more robust to waveguide dimensional variations, one can optimize the waveguide dimensions [3] or use the less confined TM-polarization [4]. The drawback of using the TM-polarization is the larger bending radius (smaller FSR) but also a higher power is needed to tune the resonances to an initially unknown incoming laser grid, owing to the lower confinement in the silicon core. In this paper, we demonstrate a TE-polarized 4-channel ring-resonator based demultiplexer with collective thermal tuning. The receiver grid can be tuned to the incoming laser grid and is robust to variations in channel spacing due to waveguide dimensional variations. A comparison is made between a firstorder and second-order MRRs regarding robustness towards resonance shifts and the resulting variation on filter crosstalk and insertion loss. Furthermore, we characterize the collective thermal tuning.

\section{EXPERIMENT}

\section{A. Design}

The demultiplexer consists of 4 cascaded filters implemented with first and second order MRRs. The channel spacing is designed to fit a grid spacing of $300 \mathrm{GHz}(2.4 \mathrm{~nm})$ and a FSR of $12 \mathrm{~nm}$. The rings are implemented with a $9 \mu \mathrm{m}$ coupling length and a $5 \mu \mathrm{m}$ radius. A small increase in circumference of the ring $(75 \mathrm{~nm})$ is used to establish the shift in resonance wavelength according to the defined channel spacing. The filters are designed to have a crosstalk lower than $-20 \mathrm{~dB}$ towards their neighboring channel. This results in a busring waveguide power coupling of 0.08 for the first order MRR (design 1) and a bus-ring and ring-ring waveguide power coupling of respectively 0.32 and 0.04 for the second order MRR (design 2). Using a 450nm wide and 220nm high waveguide dimension with oxide upper cladding for both the 
bus and ring one ends up with bus-ring gap of $295 \mathrm{~nm}$ for design 1 and bus-ring and ring-ring gap of respectively $205 \mathrm{~nm}$ and $340 \mathrm{~nm}$ for design 2 .

\section{B. Fabrication}

The WDM filter was fabricated on a $200-\mathrm{mm}$ SOI wafer with 2- $\mu \mathrm{m}$ buried oxide and 220-nm top c-Si layer using a subset of processing modules from imec's Silicon-Photonics Platform (iSiPP). Two silicon patterning steps were carried out in which respectively $70 \mathrm{~nm}$ and $220 \mathrm{~nm}$ of the c-Si layer were locally etched to define fiber-grating couplers as well as the ring filter and the strip access waveguides. The heaters were implemented as highly p-type doped, 1- $\mu \mathrm{m}$-wide silicon resistors, located at $1.25 \mu \mathrm{m}$ away from the inner edge of the ring waveguides. Local silicide and a CMOS-like tungsten/copper back-end are used to contact the heaters.

An illustration of 4-channel demultiplexer with collective heaters is given in Figure 1.

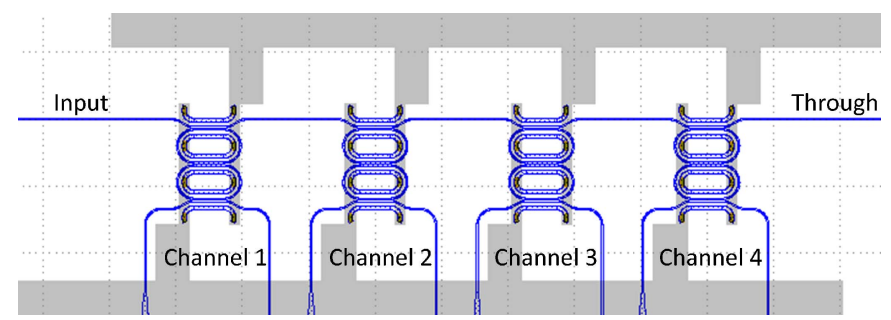

Figure 1: A 4-channel demultiplexer using $2^{\text {nd }}$ order MRRs

\section{Results}

Both designs are characterized using a tunable laser with a resolution of $10 \mathrm{pm}$. In Figure 2, one can see a typical spectral response of design 1 with first order MRRs and in Figure 3, the spectral response of design 2 with second order MRRs. The $3 \mathrm{~dB}$ bandwidth (BW) of design $\mathrm{nr} 2$ is indeed much larger $(3 \mathrm{~dB} \mathrm{BW}=1.23 \mathrm{~nm})$ than design $\mathrm{nr} 1(3 \mathrm{~dB} \mathrm{BW}=0.43 \mathrm{~nm})$.

To test the robustness of both receivers to lock into a fixed channel grid of $2.4 \mathrm{~nm}$, we manipulate the measured responses and shift all channel resonances with a multiple of the designed channel spacing on top of each other. In this way, one easily detects variations of the resonance frequencies away from their ideal spectral position. Next, the collective tuning of the resonances of the MRRs is emulated by choosing the best position of the receiver grid that minimizes the insertion loss (IL) of the worst channel. This is illustrated in Figure 4 for a first order MRR (design 1) and in Figure 5 for a second order MRR (design 2). The worst case and mean IL and cross talk (XT) corresponding to the best position of our receiver grid can then be calculated over all channels, as is illustrated in the figure. Comparing Figure 4 and Figure 5 indicates that the broader BW makes the second order filter more robust towards insertion loss and crosstalk variations.

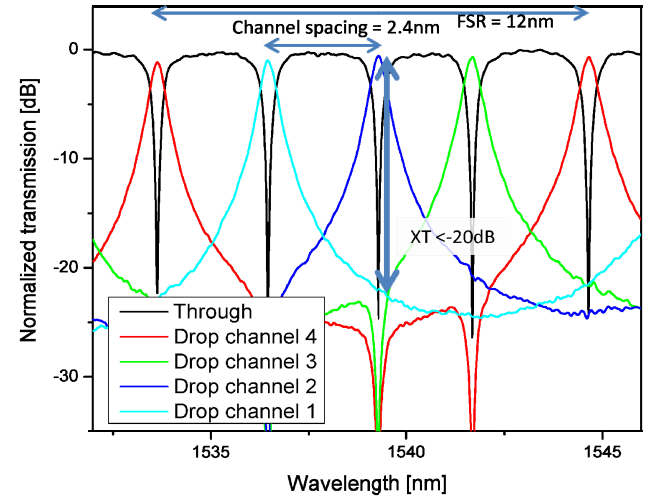

Figure 2: Spectral response of design 1 with $1^{\text {st }}$ order MRRs. The concept of FSR, channel spacing and crosstalk is explained.

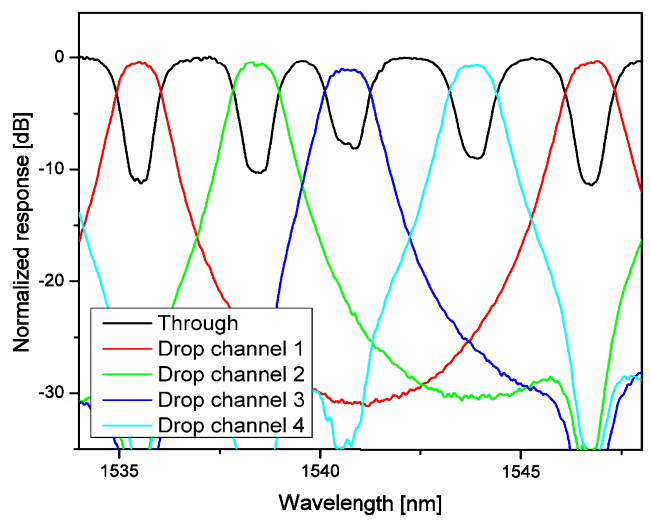

Figure 3: Spectral response of design 2 with $2^{\text {nd }}$ order MRRs.

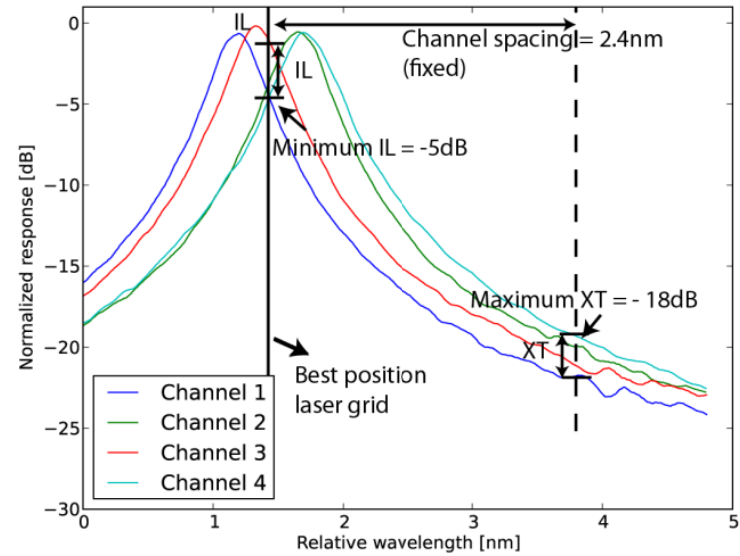

Figure 4: Insertion loss (IL) and crosstalk (XT) robustness analysis for a $1^{\text {st }}$ order MRR (design 1) 


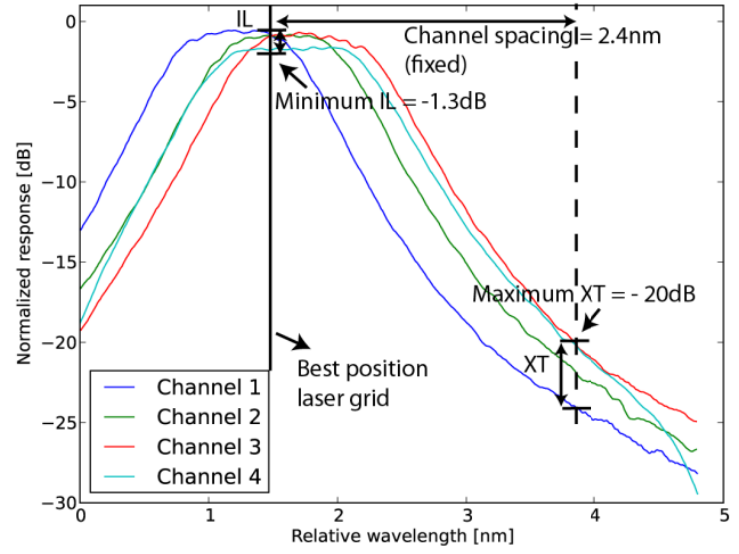

Figure 5: IL and XT robustness for a $2^{\text {nd }}$ order MRR (design 2)

To compare both designs at wafer-scale, the same calculation is made on the measurements of exactly the same designs but fabricated on different positions on the same wafer. In total, 19 measurements are collected for each design and statistically compared. The following table gives an overview of the results, comparing the IL and XT of both designs are compared both for an individual channel (denoted as "filter"), as well as for being exploited in a fixed grid with 4 channels (denoted as "grid"). The difference comes from the fact that the optimal position of the receiver grid for the whole demultiplexer is usually not the best operation wavelength when being used as a stand-alone filter.

\begin{tabular}{|c|c|c|c|c|}
\hline Design & & mean(mean) [dB] & mean(WCS) [dB] & mean(std) [dB] \\
\hline 1 & IL filter & -0.53 & -0.69 & 0.13 \\
\hline & IL grid & -2.42 & -4.5 & 1.45 \\
\hline & XT filter & -19.7 & -19.2 & 0.31 \\
\hline & XT grid & -17.7 & -15.9 & 1.39 \\
\hline \multicolumn{5}{|r}{} \\
\hline 2 & IL filter & -0.62 & -1.11 & 0.35 \\
\hline & IL grid & -0.86 & -1.33 & 0.38 \\
\hline & XT filter & -21.1 & -20.3 & 0.61 \\
\hline & XT grid & -20.8 & -18.9 & 1.42 \\
\hline
\end{tabular}

The first column describes the mean value over all positions of the mean insertion loss over all channels in one design. The second column gives the mean value of the worst case scenario (WCS), i.e. the minimum IL and the maximum $\mathrm{XT}$ such as denoted in Figure 4 and Figure 5. The difference between "stand alone" and "grid" is clear: in average, the IL of the worst channel is degraded by nearly $4 \mathrm{~dB}$ for a first order MRR (design 1) and only $0.22 \mathrm{~dB}$ for a second order MRR (design 2). The WCS XT is on average degraded by $3.3 \mathrm{~dB}$ for design 1 and $1.4 \mathrm{~dB}$ for design 2 . On average, design 2 thus compensates for IL variations and wins $1.56 \mathrm{~dB}$ power over design 1. Also, design 2 has a superior XT in the order of an average improvement of $3.1 \mathrm{~dB}$.

To finalize, we demonstrate our collective thermal heater covering all 4 channels such as illustrated in Figure 1. This is shown in Figure 6, where the resonances are shifted by a whole FSR. For clarity only one channel is plotted. The thermal tuning efficiency is $0.025 \mathrm{~nm} / \mathrm{mW}$ to tune all 4 channels collectively.

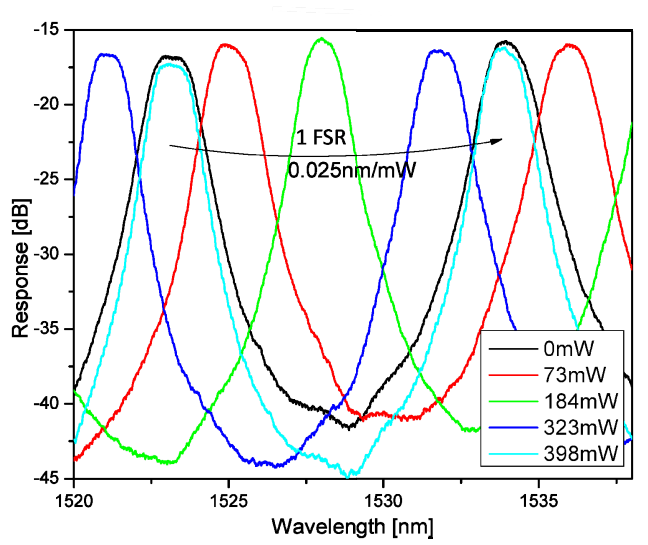

Figure 6: Collectively thermal tuning with an efficiency of $0.025 \mathrm{~nm} / \mathrm{mW}$ for a 4-channel demultiplexer. (Figure shows only 1 out of 4 channels)

\section{CONCLUSION}

A comparison is made between first- and second-order filters regarding the robustness of the filter response to waferscale fabrication variations for a 4-channel WDM demultiplexing filter. Silicon heaters are implemented to collectively tune the filter drop channels to the wavelength grid of a WDM laser source. After collective tuning, the 4channel second-order filter exhibits an average channel insertion loss of $-0.8 \mathrm{~dB}$ and an average cross talk of $-20.8 \mathrm{~dB}$, an improvement of respectively $1.56 \mathrm{~dB}$ and $-3.1 \mathrm{~dB}$ over firstorder 4-channel filters. Such a fabrication-tolerant filter with collective tuning is a key element for the implementation of a robust WDM receiver with simple active thermal control.

\section{REFERENCES}

[1] Miller, B. D. A. B. (2009). Device Requirements for Optical Interconnects to Silicon Chips. Proc. IEEE (Vol. 97, pp. 1166 - 1185).

[2] Thourhout, D. V., Spuesens, T., Kumar, S., Member, S., Liu, L., Kumar, R., Morthier, G., et al. (2010). Nanophotonic Devices for Optical Interconnect. IEEE Journal of Selected Topics in Quantum Electronics, 16(5), 1363-1375.

[3] Selvaraja, S. K., Bogaerts, W., Dumon, P., Thourhout, D. V., \& Baets, R. (2010). Subnanometer Linewidth Uniformity in Silicon Nanophotonic Waveguide Devices Using CMOS Fabrication Technology. IEEE Journal of Selected Topics in Quantum Electronics, 16(1), 316 - 324.

[4] Park, S., Kim, K.-joong, Kim, I.-gyoo, \& Kim, G. (2011). Si micro-ring MUX / DeMUX WDM filters. Optics Express, 19(14), 14627-14633.

[5] Bogaerts, W., Selvaraja, S. K., Dumon, P., Brouckaert, J., De Vos, K., Van Thourhout, D., \& Baets, R. (2010). Silicon-on-Insulator Spectral Filters Fabricated With CMOS Technology. IEEE Journal of Selected Topics in Quantum Electronics, 16(1), 33-44.

[6] Little, B. E., Chu, S. T., Absil, P. P., Hryniewicz, J. V., Johnson, F. G., Seiferth, F., Gill, D., et al. (2004). Very High-Order Microring Resonator Filters for WDM Applications. IEEE Photonics Technology Letters, 16(10), 2263-2265.

[7] Xiao, S., Khan, M. H., Shen, H., \& Qi, M. (2007). Multiple-channel silicon micro-resonator based filters for WDM applications filters for WDM applications. Optics Express, 15(12), 7489-7498.

[8] Zheng, X., Shubin, I., Li, G., Pinguet, T., Mekis, A., Yao, J., Thacker, H., et al. (2010). A tunable $1 \times 4$ silicon CMOS photonic wavelength multiplexer/demultiplexer for dense optical interconnects. Optics express, 18(5), 5151-60. 